\title{
La forza visiva nell'opera di Cesare Pavese: fra scrittura e immagine
}

\author{
ELISA MARTÍNEZ GARRIDO \\ Universidad Complutense de Madrid \\ elimarti@filol.ucm.es
}

\section{Riassunto}

Il presente saggio studia l'importanza decisiva che l'immagine-simbolo svolge all'interno dell'opera pavesiana, fondamentalmente in Feria d'agosto e ne La luna e i falò. In questi due testi di Cesare Pavese ci troviamo con delle chiare ekphrasis pittoriche che contribuendo alla semplicità stilistica della prosa pavesiana ci parlano della grande allusività poetica dei suoi testi più lirici ed elegiaci.

Parole chiave: Scrittura, immagine, simbolo, ekphrasis, poesia, pittura, Pavese.

The visual power in Cesare Pavese's works: betheen writting and image

\begin{abstract}
This essay studies the decisive importance that the symbol-image developes inside the Cesare Pavese's works, specially in Feria d'agosto and La luna e i falo. In both texts we find a clare pictorial ekphrasis which tell us about the poetical allusivity of his more liryc and elegiac novels, an evident contribution to the stilistic simplicity of Pavese's prose.
\end{abstract}

Key words: Writting, image, symbol, ekphrasis, poetry, painting, Pavese.

Martínez Garrido, Elisa. 2011. La forza visiva nell'opera di Cesare Pavese: fra scrittura e immagine. Cuadernos de Filología Italiana, nº extraordinario: págs. 233-253.

Sumario: 1. Riflessioni generali sulla scrittura e l'immagine in Pavese 2. La forza poetica dell'immagine figurativa in Lavorare stanca e in Feria d'agosto 3. La sensualità visiva ne La luna e i falò 4. I paesaggi dipinti de La luna e i falò 5. Pittura d'epoca e figure femminili de La luna e i falò 6. A modo di «bozzetti» conclusivi. Bibliografia. 


\section{Riflessioni generali sulla scrittura e l'immagine in Pavese}

Cesare Pavese nell'ormai lontano 1934, nel Mestiere di poeta diceva:

Le parole stesse che ho usato lasciano intendere che a fondamento di questa mia fantasia sta una commozione pittorica; e infatti poco prima di dar mano a Paesaggio avevo veduto e invidiato certi nuovi quadretti dell'amico pittore stupefacenti per evidenza di colore e sapienza di costruzione. Ma, qualunque lo stimolo, la novità di quel tentativo è ora per me ben chiara: avevo scoperto l'immagine (Pavese 1966: 199).

Sulla stessa linea, lo scrittore, nel periodo dal 1934 al '38, si rende conto della potenza creatrice che l'immagine visiva ha avuto nella genesi artistica della sua poesia-racconto. Nel Mestiere di vivere, le pagine dedicate alle riflessioni metapoetiche di questi anni sono gremite di importanti commenti estetici riguardanti la forza evocativa dell'immagine. Questa ci si mostra come il simbolo primordiale di una realtà primigenia; di una realtà sensoriale che, tramite la sua potenza visiva, ci porta fino a qualcosa d' "altro", fino alla poesia in se stessa, fino alla poesia del vero che è, in quanto tale, mito, sentimento tragico, ma anche ricerca del sublime.

Ricordiamo ancora le parole dell'autore:

Che le immagini che si scambiano e si illuminano, siano il progresso di ciascuna poesia è uno stato di fatto (24 nov 1935; Pavese 2000: 19-20).

I miei ritratti furono quadri, non drammi. Mi sono fissato su figure e le ho tanto rimuginate e contemplate, da riprodurre una trasfigurazione soddisfatta (22 aprile 1936; Pavese 2000: 34-35).

Tutto quanto potevo concedere alla 'poesia pura' risulta dalla unificazione estatica di ogni poesia nell'attimo contemplativo...tutto si risolverà in un'illuminazione accesa dai vari pensieri e dalle sensazioni intrecciate. L'immagine-racconto era questo soltanto (20, nov. 1937; Pavese 2000: 54).

Da queste citazioni sembra più che evidente che l'esercizio pittorico fosse a Pavese molto famigliare. Per lui esisteva, quindi, un forte legame fra l'arte figurativa e la sua plasmazione verbale nel testo poetico. Come si sa, lo scrittore, a Torino, viveva le sue esperienze artistiche e personali insieme agli amici pittori, e in modo particolare insieme a Mario Sturani ${ }^{1}$.

Non soltanto la sperimentazione pittorica dell'espressionismo e del futurismo fu decisiva nella loro formazione estetica, ma essi stessi parlano, con profondo rispetto ed ammirazione, del peso determinante che il chiaroscuro dell'impressionismo aveva avuto nella rappresentazione del paesaggio e nel rafforzamento visivo e sensuale del colore. Massimo Mila, l'amico musicista di Pavese, ci dice in proposito:

1 Il capitolo IV de La bella estate ci offre un esempio paradigmatico dell'importanza determinante dell'esperienza della pittura nella vita dei giovani artisti del gruppo. Per ulteriori informazioni sul legame di Cesare Pavese con gli amici pittori di Torino rimando a (Lamberti 2007: 37-47). 
Ma quanto ci aveva arricchiti la lezione dell'impressionismo, la rivelazione dei pregi del non-finito! E infine non si dimentichi la presenza nel nostro gruppo di un pittore come Mario Sturani, il cui studio era il quartiere generale della 'banda': ogni suo lavoro veniva sospetto al più estemporaneo giudizio collettivo nell'esercizio di una critica quanto mai confidenziale (Mila 1985).

Sia le parole dell'autore sia quelle di Mila si mostrano, di conseguenza, chiavi per l'ulteriore consistenza di questo lavoro, dedicato alla forza poetica della visività dei testi pavesiani, soprattutto di quelli rintracciabili all'interno de La luna e $i$ falò. Perchè se è vero che la potenza dell'immagine si trova in tutto Pavese, nel romanzo del '50, essa raggiunge sicuramente il suo acme definitivo.

Partendo da queste premesse, in questo saggio, si è tentato di studiare alcune delle «visioni» memoriali del romanzo, le quali, agendo da chiare ekphrasis ${ }^{2}$, si muovono esteticamente in sintonia con le riflessioni artistiche pavesiane sulla pittura; alcune delle più rilevanti (lontane dall'espressionismo e dal futurismo) sono piuttosto affini ad una linea impressionista, simbolista e decadente. Le pavesiane, sono, quindi, ekphrasis alla ricerca del mito e anche della pura bellezza e della pura sensualità, sempre concepite all'interno del gioco dei contrasti che la luce e l'ombra offrono in quanto realtà basica contrastiva.

Come si è appena detto, l'opera di Pavese, da Lavorare stanca (momento in cui nasce l'intuizione folgorante dell'immagine-poesia-racconto) fino a La luna e i falò (culmine della sua scrittura poetica, e sintesi artistica e della propria realtà esistenziale) è satura di paesaggi, di ritratti, di studi di prospettiva, di chiaroscuri, di contrasti estetici tra il gioco dell'ombra e della luce, d'intensificazione dello spessore ermeneutico del testo, grazie alle forti valenze mitiche e simboliche potenziate dagli stessi colori scelti all'interno delle descrizioni.

Un tale fatto mette in rilievo il ruolo determinante che l'arte figurativa ha giocato all'interno dell'immaginario poetico del grande scrittore del Novecento. A parte l'esperienza personale che l'autore aveva della pittura, tramite gli amici pittori, siamo dell'avviso che l'impressionismo e il post-impressionismo siano stati un suo punto di partenza definitivo nella determinazione visiva che l'immagine occupa all'interno della sua opera; anche se Pavese, forse, non ne era del tutto consapevole, e queste visioni formavano parte della sua «memoria involontaria».

Alla base di questa prospettiva estetica e figurativa di maturità pavesiana, soggiace la potenza visiva delle stampe e degli almanacchi della sua infanzia. Sono le immagini rimaste nel suo ricordo e nel suo immaginario infantile, come i «tesori del fondo della vasca» (Pavese 2002: 101-102), quelle che agiscono dal suo inconscio immaginario allo scopo di svegliare le sue capacità memoriali ed evocative. E queste immagini presero, probabilmente, ispirazione nei quadri dei grandi maestri di pittura della seconda metà dell'Ottocento, tanto italiani quanto francesi.

2 Il termine ekphrasis in questo lavoro è stato usato nella sua più antica accezione della retorica ellenistica. Pertanto, come tentativo di raggiungere la percezione visiva tramite la parola (Lavezzi 2004). 
Ma sentiamo meglio di nuovo Pavese:

[...] si può sostenere che i simboli non radicano tanto nei suoi incontri libreschi o accademici quanto nelle mitiche e quasi elementari scoperte dell'infanzia, nei contatti umilissimi e inconsapevoli con le realtà quotidiane e domestiche che le hanno accolte al principio: non l'alta poesia, ma la fiaba, il litigio, la preghiera, non la grande pittura, ma l'almanacco e la stampa, non la scienza, ma la superstizione (Pavese 2002: 160).

Ancora lo stesso scrittore, facendo riferimento al processo d'apprendimento infantile - chiaramente segnato da Leopardi (Gioanola 2002: XXVII) - parla dell' immagine antica che nell'età fanciullesca attiva la fantasia creatrice, perché da bambini «s'impara a conoscere il mondo non -come parrebbe- con inmediato e originario contatto con le cose, ma attraverso i segni delle cose: parole, vignette, ricordi» (31 agosto 1942; Pavese 2000: 243). Ovviamente, a questo sostrato mitico infantile pavesiano, si è sovrapposta l'alta cultura, della quale l'autore è stato uno dei più grandi rappresentanti; alla popolarità della fiaba e della preghiera si è aggiunta la grande letteratura; l'umile visività della stampa è stata arricchita dalla potenza pittorica della tradizione estetica del suo tempo.

Per questo motivo, la potenza visuale delle sue immagini nelle poesie-racconti di Lavorare stanca, nelle descrizioni poetiche di Feria d'agosto, in tante delle sue narrazioni e ne La luna e i falo, in modo particolare, acquistano un'enorme carica di poesia figurativa. Pare evidente che lo scrittore fosse ben consapevole dell'importanza evocativa e simbolica che la pittura giocava all'interno della sua opera. Perché lo studio delle immagini, del colore, della luce diventa, quindi, nei testi pavesiani, simbolo e metafora personale del suo stesso immaginario artistico.

La presenza ricorrente di questi simboli e di queste metafore ossessive, come era già stato osservato da Giorgio Bàrberi-Squarotti (Bàrberi Squarotti 1964: 170), porta, di conseguenza, la scrittura pavesiana verso la fuga nella stessa metafora. Seguendo la traccia del critico piemontese, siamo dell'avviso che questa «fuga nella metafora» non sia altro che una nuova forma di affrontare la poeticità visiva della letteratura di Pavese.

Ma è da precisare che la potenza visiva della sua scrittura, e la sua marcatissima tendenza verso l'ekphrasis, non è soltanto una questione di contenuto, anzi. Si potrebbe affermare, invece, che le descrizioni (o forse meglio le non-descrizioni) pittoriche di Pavese, scarne e semplici, se da una parte ci si mostrano come una sua scelta stilistica (condizionata dalla voluta ed apparente "rusticità" della sua prosa), dall'altra sono le responsabili dirette della marcata e forte allusività simbolica della sua opera. Così, dunque, il mistero e la tensione tragica della scrittura pavesiana agiscono simbolicamente fin dall'inizio dei suoi testi, ma in modo nascosto. Le immagini visive diventano, quindi, i pilastri fondamentali dell'implicito pavesiano. Queste dicono senza dire, in un crescendo tragico e estetico annunciato fin dall'inizio dell'opera.

Possiamo affermare allora che la potenza figurativa delle immagini dello scrittore apre la strada verso la ricchezza polisemica della sua letteratura. Cioè, lo stile 
visivo pavesiano, in stretto rapporto con la psicologia del profondo e con il pensiero mitico-antropologico, fa sì che molte delle sue opere siano vere e proprie elaborazioni ekphrastiche. Queste ci rimandano ad un immaginario visivo, tanto popolare quanto colto, appartenente, solo in parte, alle esperienze figurative del secondo Ottocento e del primo Novecento.

Per questo motivo, data la forza visiva svolta dalla pittura nella letteratura di Pavese, bisogna anche ricordare che non è casuale che di tutte le stagioni dell'anno, quella pavesiana per eccellenza sia l'estate; soprattutto per il Pavese langarolo, perché, invece, quello torinese dipinge anche l'inverno ${ }^{3}$. Santo Stefano Belbo era il «loco natio» dove ritornava lo scrittore per le vacanze. La Langa, quindi, rappresenta il tempo dell'estate mitica in campagna, il momento dei giochi e delle scoperte infantili e adolescenziali.

L'estate langarola inoltre raddoppia la forza visiva dei chiaroscuri, la potenza dei colori, legati questi a tutta la simbologia mitica del rituale agrario e festivo. Pavese, dunque, da vero poeta, fra il verde delle sue colline, traduce in parole le immagini visive delle proprie antiche estati infantili. In questo modo, le «visioni» memoriali delle proprie vileggiature dell'adolescenza diventano testo poetico e pittorico allo stesso tempo, pura poesia e pura creatività; non carente, però, di una marcata tensione tragica, proveniente inesorabilmente dalla violenza, dalla sessualità e dalla storia.

Non dobbiamo dimenticare che lo stesso autore era ben consapevole della forza immaginaria e del valore simbolico e mitico del colore, tra cui indubbiamente dobbiamo includere il chiaroscuro. Scrive l'autore nel Diario:

Un giardino tropicale in mezzo alla neve. Magnolie/abeti, tassi, cipressi, limoncelle - verdi cupi, metallici bronzei contro il cielo azzurro. Ma ciò che li rialza di più, è il muro rosso mattone della scuderia colonica. $\mathrm{Ci}$ sono tutti i più intensi colori naturali: verde, azzurro, rosso, candido. Colpisce l'insolito o c'è una segreta virtù in queste qualità pure?

È facile ai colori diventare simboli. Sono la qualità più vistosa degli oggetti ma non sono gli oggetti. Ricordando che hai detto una volta che il mito vive negli epiteti, i colori sarebbero gli epiteti delle cose. Creazione pura.

[...] si può dire di tutte le sensazioni pure: sono simboli che tendono a sostituire la natura (28 gennaio, 1945; Pavese 2000: 296-97).

È grazie alla precisa e sottile elaborazione immaginaria della sacralità spaziale propria dei «luoghi unici» (Pavese 2002: 149), grazie allo studio sensoriale della luce e del colore, grazie all'allusività che le connotazioni ultime della luminosità e dell'oscurità comportano; insomma, grazie alla poderosa visività dei testi pavesiani, che lo scrittore italiano diventa, anche, un grande poeta, un autore classico e di grande modernità, allo stesso tempo.

3 A parte La bella estate si pensi anche a Tra donne sole, dove appare anche l'inverno, di fatto il romanzo inizia con le nevi di gennaio (Pavese 2008: 449). 
Possiamo concludere questa prima parte del lavoro rifacendoci alle riflessioni di Pier Vincenzo Mengaldo sul rapporto fra la poesia e l'arte pittorica. Secondo lo studioso milanese, è lecito affermare che i veri scrittori sono quelli capaci di arrivare all'arte figurativa e di tradurre con le parole la forza e la potenza simbolica dello spazio, del colore e della luce (e Mengaldo ci parla dei grandi scrittori dell'Ottocento francese: Flaubert, Baudelaire, Mallarmé) (Mengaldo 2005: 70-75). Pavese, di conseguenza, a partire da questi parametri critici, grazie a questa stessa realtà di captazione visuale della sua scrittura, può essere considerato un classico del Novecento; in questa sua potenza di visione, pertanto, rimane nascosta tutta la forza del non detto pavesiano e il mistero poetico e tragico della sua opera. Grazie all'elaborazione ekphrastica di Pavese, la sua letteratura suggerisce molto di più di quanto esplicitamente dice.

\section{La forza poetica dell'immagine figurativa in Lavorare stanca $\mathbf{e}$ in Feria d'agosto}

Come abbiamo già ripetuto precedentemente, l'opera di Pavese, fin dalla raccolta poetica del '36, ricrea vere e proprie ekphrasis. Questa sua visività poetica, originariamente propria, in una sua seconda fase di elaborazione formale, ci rimanda ai grandi modelli della poesia italiana e non italiana della sua formazione: Gozzano, Whitman, Baudelaire e Melville (Guglielminetti 1998: IX-XII; Mondo 1964: 3-21).

In questa stessa linea di visività figurativa, tutta la serie dei Paesaggi, I, II, III, IV, V, VI, VII, VIII, Grappa a settembre, Città in campagna, Estate ( Pavese 1998: 12, 22, 23, 29, 32, 43, 53, 63, 81), o Le maestrine (oggi edito anche nei Racconti) (Pavese 2006: 360-362); si contraddistinguono per la forza visiva di un'immagine essenziale, dipinta come esercizio paesaggistico o come ritratto. In questi casi, siamo davanti a quadri fatti di parole, nati in apparenza solo da un'estetica realista, stampe che hanno catturato la poesia immanente del vero e dell'epopea del quotidiano.

Nelle opere posteriori, soprattutto in Feria d'agosto o ne La luna e i falò, lo scrittore rinforza ulteriormente la potenza sensoriale e visiva dei suoi testi. Pavese, dunque, dopo il 1946, grazie al valore simbolico puro del colore e della luce, rinforza l'allusività suggestiva dei suoi componimenti, e, raddoppiando la sua capacità figurativa e mitica nello studio del vero, arriva all'estasi epifanica.

Sappiamo che in noi l'immagine inaspettata non ha avuto inizio: dunque la scelta è avvenuta di là dalla nostra coscienza, di là dai nostri giorni e concetti; essa si ripete ogni volta, sul piano dell'essere, per grazia, per ispirazione, per estasi insomma (Pavese: 2002: 157).

La realtà che si rivede nella sua opera, dunque, con gli occhi dell' antico fanciullo, ci mostra come una vera ed autentica rivelazione. Tramite le valenze mitiche e la ricchezza ermeneutica delle immagini-simbolo pavesiane, l'autore tenta di lasciare parlare il silenzio con un significante altro; si tratta di un linguaggio lontano dalla 
doppia codificazione simbolica. Pavese rappresenta così il muto linguaggio delle cose e lo traduce in una lingua superiore, perché in ogni opera d'arte appare sempre qualcosa che esplicitamente non c'è (Adorno 1970: 119).

Grazie al valore evocativo e simbolico dell'ekphrasis, lo scrittore racconta, dunque, l'assoluto, che per lui è sia il tragico che il bello in sè stesso. Di conseguenza, se come dice Elio Gioanola, (2002: xix) la genesi della poesia appartiene al pre-linguaggio, questa è indicibile. Per questa ragione, Pavese, a partire dal 1946, tenta di presentarla soltanto attraverso le immagini e le evocazioni mitiche e simboliche dei suoi testi; cioè, attraverso il linguaggio figurativo. Questo gli consente di esprimere quello che è inesprimibile: la commozione sublime e tragica del poetare.

È chiaro che con queste affermazioni siamo già pienamente entrati in Feria di agosto, opera che, in un certo senso, potrebbe essere intesa come un manifesto della scrittura poetica di Pavese. All'interno della raccolta, in stretto rapporto all'importanza fenomenologica della figuratività, si intensifica la presenza lessicale del campo semantico dello sguardo; vedere, guardare, sguardo, visione: «Vorrei essere sempre disposto a respirare, a vedere. Vedere, vedere sempre mi basterebbe» (Pavese 2002: 109), perché nell'atto contemplativo del vedere si nasconde l'essenza stessa della poesia e dell' "altro". Pavese ha già concepito, quindi, l'attimo estatico e contemplativo, che è sostanzialmente memoria visuale di pura poesia (Pavese 2000: 157).

Perciò, in tanti racconti e saggi di Feria d'agosto si intensifica considerevolmente il rapporto oppositivo luce-ombra, in stretta corrispondenza con la dialettica spaziale e simbolica tra il fuori e il dentro. Si pensi a L'adolescenza (Pavese 2002: 161) o a L'estate, dove il gioco dei chiaroscuri e dei colori crea una vera e propia rete pittorica. Basti ricordarne soltanto l'incipit: «Di tutta l'estate che trascorsi nella città semivuota non so proprio che dire. Se chiudo gli occhi, ecco che l'ombra ha ripreso la sua funzione di freschezza, e le vie sono appunto questo, ombra e luce, in paesaggio alternato che investe e divora» (Pavese 2002: 106).

Tramite questa intersezione dello sguardo contemplativo, lo spazio cerniera della finestra diventa anche nell'opera pavesiana il punto morfologico centrale che consente l'unione di entrambi i luoghi. La finestra potenzia ulteriormente lo stesso contrasto visivo in sé stesso. In questo senso, Vocazione non è se non una visione artistica del mondo attraverso la finestra ${ }^{4}$.

Ricordo quanti papaveri si vedevano dalla finestra nella campagna, e quelli non me li ero certo sognati. Colori così vivi non si sognano [...] Ma quei papaveri $[\ldots]$ spuntavano sul rialto, dentro la finestra [...] Questo senso di fiducia mi è abbastanza famigliare, e mi prende ogni volta che da un luogo chiuso do un'occhiata al cielo, alle piante, all'aria [...] (Pavese 2002: 109).

${ }^{4}$ La finestra è uno spazio simbolo chiave nella creazione pavesiana. Già ne $\mathrm{Il}$ carcere la visione della finestra coi geranei, aperta sul mare, è uno spazio di grande suggestione estetica, che annuncia, dall'implicito, la figura femminile della 'capra' (Pavese 2008: 148). Per ulteriori sviluppi cfr. Basile (2003). 
Ma dobbiamo ricordare anche che la vista (interiorizzata attraverso il ricordo) gioca anche un ruolo decisivo nel saggio Del mito, del simbolo e d'altro, dove l'autore rende conto della sua profonda commozione estetica davanti alla realtà, da cui nasce il desiderio sempre insoddisfatto dell'atemporale, del ritorno alle origini; cioè, al mistero della poesia, che in parte proviene dall' «occhio netto»:

Non si parla qui della poesia, che è sempre possibile, specie quando la si vuole, e in definitiva dipende soltanto dalla pazienza e dall'occhio netto. Ma di quell'immagine o ispirazione centrale, formalmente inconfondibile cui la fantasia di ciascun creatore tende inconsciamente a tornare e che più lo scalda con la sua onnipresenza misteriosa (Pavese 2002: 154).

Tra Feria d'agosto e La luna e i falo, Pavese ha portato avanti tutto l'approfondimento sul mito e la tragedia, il cui indiscutibile acme intellettuale è Dialoghi con Leucò. Ma nella chiusura della sua carriera letteraria, che segna anche la conclusione della propria vita, l'autore fa ancora una volta un viaggio a ritroso; un viaggio doppio, al termine del quale si ritrova indubbiamente con un nuovo e rovesciato passaggio estetico ed esistenziale, questa volta, dal mito tragico, il poeta approda nella pura poesia visuale (Bossetti 1960: 175).

\section{La sensualità visiva ne La luna e i falò}

La capacità evocativa della poesia di Pavese, insieme allo spessore simbolico dei racconti-saggi di Feria d'agosto, ci conduce chiaramente fino a La luna e i falò, il romanzo di Pavese nato come «bozzetto nudo a Santo Stefano Belbo» nella vacanza di giugno de 1949 (Pavese 1966a: 498). Il 22 giugno dello stesso anno, l'autore scrive su quest'ultima villeggiatura: «c'è stato soltanto sole e acqua» (Pavese 1966a: ivi). Siamo arrivati in questo modo, tramite la commozione sensibile della luce estiva e del suo riverbero sull'acqua, ai due dei grandi motori visivi della creatività poetica e simbolica pavesiana.

D'altra parte, non si deve dimenticare che, all'interno del romanzo del 1950, è la potenza creatrice dello sguardo dell'io narrante a strutturare tutto il viaggio memoriale del protagonista ${ }^{5}$, un chiaro doppio dell'esperienza poetica dello stesso scrittore. Non è casuale, pertanto, che La luna sia stato il libro che Pavese si «portava dentro da tanto tempo» (lettera del 23 maggio 1950; Pavese 1966b: 532), quello che ha avuto la sua genesi, in parte, nella Divina Commedia, e fondamentalmente in tutta la polisemia irrazionale e mitopoetica che comporta il sintagma dantesco «mirabile visione» (17 luglio 1949; Pavese 1966: 489). Questo stesso sintagma, usato dall'autore, mette a fuoco l'importanza decisiva della contemplazione visiva all'interno del testo; quella che parte sempre, insomma, da uno sguardo irrazionale, fantastico e memoriale, da un mirare contemplativo e ammirabile, dal quadro stesso, che non è altro che il congelamento del tempo, l'attimo statico, la ekphrasis poetica.

${ }^{5}$ Per Diderot, le visioni poetiche che provengono dalla realtà seguono sempre lo schema del viaggio o della passeggiata di chi può, dallo stato contemplativo puro del solitario, arrivare alla poesia (Mengaldo 2005: 59). 
Dopo quanto è stato detto fin qui, in un certo senso, si potrebbe affermare che ne La luna e i falò, Pavese percorre la strada contraria a quella fatta in Lavorare stan$c a$. Malgrado la vicinanza che l'immaginario pavesiano della raccolta del '36 presenta nei confronti del romanzo del '50 (Gioanola 1971:375), le differenze fra queste due opere ci si mostrano evidentissime. Cioè, se in Lavorare stanca lo scrittore era partito dall'immagine-poesia per arrivare al racconto, ne La luna Pavese, iniziando la strada a partire dalla storia raccontata, dal dialogo e dalla tragedia, approda, soprattutto nei momenti più elegiaci del testo, alla pura poesia. Le immagini provocano così un evocare di ricordi sensitivi fondamentalmente visivi.

Ma ritorniamo al piano formale del romanzo, allo scopo di stabilire delle corrispondenze letterarie tra forma e contenuto. Come è già stato sostenuto da Gian Luigi Beccaria, ne La luna e i falo, «la prosa pavesiana diventa poesia» (Beccaria 2000: XIV). All'interno dei XXII capitoli del libro, non legati fra di loro dal filo conduttore dell'intreccio, ma giustapposti in blocchi tematici statici (alcuni dei quali di grande forza visiva), spicca, come si è già visto, la frammentarietà della prosa poetica. Questa si incentra sulla visività memoriale del protagonista, e viene continuamente intercalata fra i dialoghi di Nuto e Anguilla, dedicati alla vita e alla storia, alla violenza, alla guerra, allo sfruttamento e alla misera rurale; quella che costringe, per esempio, Vallino a diventare personaggio tragico e sottomesso alla legge inesorabile del suo destino di dolore e di morte.

Ed è proprio qui, in queste scene «inframmezzate da inserti lirici» (Beccaria 2000: XIV) (dove la frammentarietà compositiva de La luna e i falò agisce nel modo più evidente), che la visività ekphrastica di Pavese raggiunge il suo maggiore climax poetico. D'altra parte sono le descrizioni della natura, delle figure e delle cose di un altro tempo, quelle che veramente stabiliscono, all'interno del testo, il reale filo conduttore fra le diverse scene e diversi motivi narrativi del romanzo. La concinnitas de La Luna, lo stile «secco del libro» (Beccaria 2000: XXV) solo in apparenza, sono dovuti, a mio avviso, non soltanto alla caratteristica ricerca pavesiana dello «stile semplice», bensì alla frammentarietà legata ad ogni ekphrasis poetica.

E l'ekphrasis, dunque, ad imporre anche al romanzo la frammentarietà visiva $\mathrm{e}$ poetica di questo testo elegiaco, il quale si muove nell'impossibile recherche $d u$ temps retrouvé e nella memoria malinconica della rivisitazione del ricordo della "Bellezza Femminile". Non dimentichiamo che il desiderio di Anguilla di rivedere le ragazze si mostra fin dall'inizio dell'opera come il principale scopo esistenziale del viaggio a ritroso del protagonista in Langa.

Potevo spiegare a qualcuno che quel che cercavo era soltanto di vedere qualcosa che avevo già visto? Vedere dei cani, vedere dei fienili, vedere una bigoncia, una griglia, un fiore (Pavese 2008: 652) ${ }^{6}$.

${ }^{6}$ Si ricordi la famosa lettera di Pavese a Pivano del 1942: «Immagini primordiali, come dire l'albero, la casa, le vite, il sentiero, la sera, il pane, la frutta, ecc. Mi sono dischiuse in questi luoghi, anzi in questo luogo, a un certo bivio dove c'è una gran casa, con un cancello rosso che stride, con un terrazzo dove ricadeva il verderame che si dava alla pergola e io ne 
Questo rivisitare visivo dei ricordi antichi del personaggio impone alla narrazione il suo inevitabile ritmo pausato. Si tratta di un ritmo lento, pacato, statico (Gioanola 1971: 380), dove la visione-ricordo dei «luoghi unici» del passato di Anguilla diventa il vero e principale protagonista dell'opera. All'interno dei quadri statici de La luna e i falo, come sappiamo attimi contemplativi, la forza pittorica visiva dell'autore raggiunge il suo massimo poetico. Forse perché come afferma Ernest. H. Gombrich, «il simbolo visivo attira sempre chi è alla ricerca delle profonde rivelazioni» (Gombrich 1997: 41).

Nella Luna e i falò, pertanto, grazie alla vista e grazie anche all'evocazione elegiaca di quello che Anguilla rivede con gli occhi del garzone di tredici anni che vive ancora nell'uomo quarantenne, l'eternità del tempo senza tempo, del tempo memoriale, che è poesia in se stessa, si rivela come l'unica finalità esistenziale dell'io narrante-scrittore. La poesia, che è pura essenza, rappresenta la pienezza assoluta della creatività pavesiana, e ci avverte anche della propria predisposizione interiore verso la prossima ed imminente fine dell'autore ${ }^{7}$.

Molti sono i momenti ekphrastici del La luna e i falò. Adesso, però, sarà possibile solo rilevarne alcuni, principalmente quelli dedicati alla contemplazione visiva del paesaggio langarolo, e soprattutto incentreremo la nostra attenzione sulle descrizioni evocative della bellezza delle giovani de La Mora. In esse lo studio simbolico del colore, della luce e dell'acqua, insieme alla stessa scelta dei motivi narrativi e figurativi, ci parlano della classicità di Pavese e del suo impressionismo simbolista, sempre alla ricerca del sublime: tragico e bello, rustico o popolare che sia.

È chiaro che per lo scrittore il bello (come per ogni classico) può solo essere capito dalla prospettiva della tragedia e della morte. Di conseguenza, mediante le ekphrasis dedicate ne La luna alla mitificazione di Irene, di Silvia e di Santina (le tre grazie), si raggiungono la bellezza, la leggenda e il mito, inseriti tutti quanti all'interno della ciclicità naturale, della legge inesorabile delle stagioni, del tempo e della morte.

\section{I paesaggi dipinti de La luna e i falò}

Nel primo esempio analizzato, possiamo già rilevare la marcata prospettiva spaziale di un quadro della pianura del Belbo, vista dal Salto. Pavese-Anguilla descri-

avevo sempre le ginocchia sporche; e rivedere perciò questi alberi, case, viti, sentieri, ecc. mi dà un senso di straordinaria potenza fantastica, come se mi nascesse dentro, l'immagine assoluta di queste cose, come se fossi bambino che porta in questa scoperta, una ricchezza di echi, di stati, di parole, di ritorni, di fantasia insomma, che è davvero smisurata [...] Ora questo stato di aurorale verginità che mi godo, ha l'effetto di farmi soffrire, perché so che il mio mestiere è di trasformare tutto in 'poesia'» (Pavese 1966: 425-426).

7 Vorrei ricordare adesso le parole di Pavese dedicate a Costance Dowling, scritte nel Diario il 26 aprile 1950, dove Pavese identifica la donna amata con la poesia. «Certo in lei non c'è soltanto lei, ma tutta la mia vita passata [...] Lei è la poesia nel più letterale dei sensi» (Pavese 2000: 395). 
ve, dalla finestra di Nuto, gli oggetti-simbolo chiave della veduta di un paesaggio, eterno e mutevole allo stesso tempo.

La descrizione di quello che il protagonista vede è disegnata con una marcatissima volontà di separazione, di concretezza spaziale, con una delicata sensibilità di costruzione, basata sul contrasto fra le realtà orizzontali e quelle verticali. Assenti i colori dal testo, questi devono essere ricostruiti attraverso la potenza coloristica dell'immagine-simbolo, quella che attira l'attenzione visiva del lettore.

Questi discorsi li facevamo sullo stradone, o alla finestra bevendo un bicchiere, e sotto avevamo la pianura del Belbo, le alberelle che segnano quel filo d'acqua, e davanti la grossa collina di Gaminella, tutta vigne e macchie di rive (Pavese 2008: 570).

In questo primo paesaggio, deve rilevarsi un chiaro gioco di prospettiva fra le linee orizzontali, quelle del filo d'acqua, del fiume e degli alberi che seguono il suo corso (bianche e verdi data la ghiaia della riva del fiume in estate, e il colore degli alberi), e quelle verticali, rappresentate principalmente dalla poderosa presenza mitica di Gaminella.

Nel secondo brano di paesaggio, entriamo già più marcatamente nel chiaroscuro e nell'importanza del colore nella scrittura pavesiana, piena di valenze simboliche. Dobbiamo precisare, però, che, in questo secondo esempio, come in tante altre occasioni, i colori di Pavese non vengono nemmeno nominati nel testo. Al contrario l'autore presenta l'oggetto scarno e nudo, senza aggettivazione, nella sua pura essenza immaginaria. Ma, grazie a un processo di deduzione analogica per contiguità, è possibile "vederlo" chiaramente nel suo colore naturale; questo ci offre la prospettiva della figurazione visiva all'interno del brano.

Questa assenza di descrizione coloristica ci si mostra anche come un ulteriore esempio del'essenzialità della prosa de La luna. La semplicità stilistica del romanzo non priva assolutamente la scrittura pavesiana dalla forza visiva del colore-simbolo; questo è sempre presente nei suoi testi, ma ci si mostra indirettamente, da una prospettiva implicita. Cioè, i diversi colori delle realtà, visti e ricordati da Anguilla, lavorano dall'interno silenzioso della descrizione testuale, grazie a tutte le connotazioni mitiche e visive che l'oggetto-simbolo pavesiano ci mette davanti agli occhi.

Così mi misi per il prato e costeggiai la vigna, cotta dal sole. Per quanto dietro la vigna, invece dell'ombra nera dei noccioli, la costa fosse una meliga bassa, tanto che l'occhio si spaziava, quella campagna era ben minuscola, un fazzoletto (Pavese 2008: 578).

Oltre alla disposizione spaziale dell'esempio, che predilige le parti nascoste della campagna langarola, nel paragrafo selezionato, compaiono anche i colori. Abbiamo qui il verde, la luce (bianca-dorata) del sole e il giallo del mais, e, in opposizione tonale, l'ombra nera dei noccioli. Il paragone fra il pezzo di terra e il fazzoletto evoca anche un'altra possibilità coloristica, dato che i fazzoletti dei contadini di solito, all'epoca, erano di forti colori luminosi. 
Nel terzo brano analizzato, siamo davanti ad una descrizione molto simile a quel-lo già studiata in 2. Il colore verde non è stato menzionato nella descrizione, ma ne rimane implicito per la presenza delle foglie. Il rosso-nero è già contenuto nella realtà-simbolo dei rovi, e il giallo in quella della meliga. L'unico colore esplicito di questo passaggio è il rosso del salice.

Si potrebbe pensare che, essendo il rosso l'unica tinta esplicitata nel paragrafo, la sua forza visuale acquisisca qui una grande intensità simbolica rispetto alle altre diverse tonalità implicite nel quadro. Se fossimo davanti ad una reale pittura, diremmo che la sua campitura è decisamente più intensa. La forza visiva del colore rosso non potrebbe annunciare, forse, in modo implicito, date le sue valenze mitiche e simboliche, il colore dell'incendio, il fuoco che Vallino provocherà più tardi alla fine della storia? ${ }^{8}$.

Quando franammo fra le foglie grasse, i rovi e la menta del fondo, il Vallino alzò appena la testa. Stava troncando con la roncola sul capitozzo i rami rossi di salice. Come sempre mentre fuori era agosto, quaggiù faceva freddo, quasi scuro (Pavese 2008: 586).

Ad ogni modo, all'interno dell'ekphrasis studiata, il verde, il rosso, il nero e il giallo, insieme all'azzurro del cielo e dell'acqua e al bianco delle nubi, indirettamente presenti in una pittura mitica del vero, esibiscono il loro marcato valore simbolico, in quanto colori primari o secondari di base. Tra l'altro la potenza del chiaroscuro di questo brano non è esplicitata, deve anche essere dedotta, però, dalla contrapposizione lassù-fuori-agosto-sole-caldo, in opposizione a quaggiùdentro-freddo.

${ }^{8}$ Il rosso è un colore privilegiato all'interno di questo romanzo e lungo tutta la produzione letteraria di Pavese. Ne La luna, Silvia, la ragazza di fuoco, è sempre vestita di rosso. La sua morte, prodotta da un aborto, raddoppia nel personaggio femminile tutte le caratteristiche simboliche inserite di questa stessa tinta. Il rosso che accompagna il personaggio femminile parla del suo calore erotico e ci annuncia anche, allo stesso tempo, la sua fine tragica. Il rosso, data la sua doppia valenza simbolica di passione sessuale e di morte tragica, rappresenta uno dei colori ricorrenti nell'opera dello scrittore. Si ricordino anche in questo senso i racconti Primo Amore, e più concretamente la scena in cui il protagonista vede attraverso la finestra l'amore sessuale tra i due giovani. In questo momento, all'interno del quadro spicca, in modo molto intenso, il fazzoletto rosso dell'uomo che si congiunge alla sorella dell'amico, il primo amore dell'io narrante (Pavese 2002: 63). Questo oggetto rosso non parla, a mio avviso, unicamente del realismo pavesiano. Non si tratta di una descrizione realista dei capi d'abbigliamento della campagna langarola all'inizio del Novecento. Il rosso forte del fazzoletto del giovane, in chiaro contrasto tonale con il colore giallo del pomeriggio estivo in cui ha luogo la scena e con il dorato della paglia dove si incontrano gli amanti, diventa un puro simbolo, tanto del fuoco sessuale del personaggio quanto del caldo d'agosto. Nella stessa linea simbolica, bisogna ricordare che il vestito che la protagonista del racconto La giacchetta di cuoio indossa proprio prima del suo incontro sessuale con l'amante è anche rosso. Anche qui il colore agisce come annuncio narrativo della sua inmediata e tragica morte (Pavese 2002: 37). 


\section{Pittura d'epoca e figure femminili de La luna e i falò}

Gli altri esempi che intendo analizzare in questo lavoro appartengono al mondo de La Mora e alle sue ragazze. La prima pittura scelta corrisponde ad una descrizione del cortile e del giardino della casa di sor Matteo. La fotografia di questi due spazi domestici funziona, in un certo modo, da quadro d'epoca, come la stampa di un tempo passato (quello dell'adolescenza di Anguilla), che non esiste più nel momento in cui il protagonista ritorna in paese.

A me piaceva quel cortile così grande - ci si stava in tanti e nessuno ti cercava- e poi era vicino allo stradone, sotto il Salto. Tante facce nuove, la carrozza, il cavallo, le finestre con le tendine. Fu la prima volta che vidi dei fiori, dei veri fiori, come quelli che c'erano in chiesa. Sotto i tigli, dalla parte del cancello, c'era il giardino, pieno di zinie, di gigli, di stelline, di dalie - capii che i fiori sono una pianta come la frutta - facevano il fiore invece del frutto e si raccoglievano, servivano alle figlie, che uscivano col parasole e quando stavano in casa li aggiustavano nei vasi (Pavese 2008: 614-615).

Siamo davanti a due spazi aperti: il cortile e il giardino. In essi si trovano gli oggetti-simbolo di un tempo perduto: la carrozza e il cavallo. Questi oggetti-simbolo sono anche il segno della famiglia benestante di allora e creano una rete associativa forte insieme ad alcuni dei marchi caratteristici della femminilità di provincia: le finestre con le tendine, e soprattutto i parasoli e i fiori.

I fiori, insieme ai frutti, come la collina-mammella onnipresente in Pavese, creano nel romanzo una rete simbolica molto produttiva, tanto all'interno dell'opera del 1950 quanto in altri suoi testi. La sensualità del profumo floreale, unita al gusto dei frutti e al loro piacere coloristico, ci porta in questi casi verso un immaginario decadente, simbolista e persino proustiano (controbilanciato dal realismo e dalla durezza tragica della realtà contadina e storica de La luna); fino alle giovani ragazze nel fior del loro tempo (Martínez Garrido 2010).

Le signorine de La Mora, come le piante, come i tigli, come i fiori e i frutti dell'estate, possiedono in loro stesse tutte le potenzialità sensoriali. Non dimentichiamo che insieme al mondo botanico, le tre sorelle (vestite tante volte con vestiti floreali) suonano il pianoforte in stanze piene di piante e di fiori; e la loro è una musica per niente simile a quella che Nuto fa per la banda (Pavese 2008: 638) ${ }^{9}$.

Anche nel primo esempio di questa seconda serie visiva, come in quelli precedenti dedicati al paesaggio, in apparenza non esiste il colore, non lo si dipinge, mai viene nominato. Siamo nuovamente davanti alla nudità scarna della - a prima vista - non-descrizione pavesiana. Eppure, il colore c'è in questa sua pittura d'epoca in un modo intensissimo. Come negli altri casi, il colore viene dato tramite l'oggetto-

${ }^{9} \mathrm{Ne}$ La luna e i falò quasi tutti i sensi sono messi in gioco: la vista, l'orecchio, il gusto. Al protagonista, però, non sarà consentito toccare né essere toccato dalle mani di quelle donne simbolo e mito della bellezza e della femminilità. 
simbolo, l'aggettivo e l'epiteto non si dicono, ma si vedono, e oserei dire che, in que-sto caso, si sentono anche. I diversi oggetti-simbolo che popolano il cortile e il giardino de La Mora non sono solo colorati: la luce del sole d'agosto ne potenzia la qualità cromatica fino alle ultime conseguenze, e in questo modo essi diventano fuoco di luce.

Per questa ragione, Pavese gioca in questo suo quadro d'epoca con le suggestioni stilistiche che gli offre l'uso della ripetizione delle parole-simbolo; quelle ricorrenti più volte all'interno di questo paragrafo. Il termine generico fiore occorre nel testo quattro volte, e quattro sono le specie floreali enumerate: zinie, gigli, stelline, dalie. Grazie a questa insistenza forte sull'iperonimo fiore e sui suoi quattro iponimi, possiamo facilmente dedurre che questi e il loro profumo, insieme ai frutti e al loro sapore, diventano, metafora centrale della fugacità del tempo e della caducità sensuale delle tre giovani donne, protagoniste assolute di questa descrizione e di gran parte del romanzo.

Il secondo esempio di questa seconda sezione appartiene già parzialmente all'interno della casa de La Mora. Nella scena, Anguilla è stato chiamato dalla signora per fare qualche commissione. Il ragazzo entra nella stanza, chiusa e oscurata, portando la luce del sole estivo nei propri occhi. Partendo da questo fatto, è facilmente deducibile che il gioco di chiaroscuri e di contrasti tonali ruota l'intera ekphrasis. Questo esempio, quindi, porta la sua forza visiva e coloristica fino alle sue ultime ed estreme conseguenze tonali in contrasto. Vediamo:

Passai il pianerottolo, attraversai due stanze scure, piene di mobili, di almanacchi, di fiori, era tutto lucido, leggero come gli specchi - io camminavo scalzo sui mattoni rossi, sbucò la signora nera, col medaglione al collo e il lenzuolo sul braccio, mi guardò i piedi.

Dal terrazzo 1'Emilia gridava: - Anguilla, vieni Anguilla.

- Mila mi chiama, balbettai.

- Va', va'- disse lei, - passa presto.

Sul terrazzo stendevano i lenzuoli lavati, e c'era il sole, e in fondo verso Canelli c'era la palazzina del Nido. C'era anche Irene, la bionda, appoggiata alla ringhiera con un asciugamano sulle spalle, che si faceva asciugare i capelli (Pavese 2008: $620)$.

Come abbiamo annunciato, indubbiamente la forza del chiaroscuro e del contrasto dei colori più vivaci attira subito l'attenzione del lettore, dato che, in questo passaggio di forte contrapposizione tonale, la luce e l'oscurità diventano potentissime. La sala è buia, oscurata, sicuramente dalle imposte, allo scopo di conservare il fresco della notte e del primo mattino, e Anguilla, entrando, si sente acciecato.

In compenso, nell'interno domestico, pieno di suggestioni sensuali, si trovano altri piccoli centri di luce e di colore, i quali, agendo dall'interno dell'ombra della stanza, raddoppiano la sensazione di contrasto visivo: i fiori e gli almanacchi del salotto, i mobili e il pavimento lucido, che, come le superfici degli specchi sono leggeri, perché riflettenti, ci si mostrano come potenti fuochi luminosi che splendono dentro il buio dell'abitazione. 
Nella prima parte del brano, la presenza stessa della signora raddoppia una volta ancora il gioco contrastivo fra la luce e l'oscurità, fra il bianco e il nero. La donna di casa, vestita di nero («la signora nera» dice testualmente il passo), porta un lenzuolo (bianco si capisce) sul braccio, e anche un medaglione, sicuramente lucente.

Ma la consapevolezza pavesiana riguardo la forza dei chiaroscuri non si ferma qui, al contrario va in crescendo lungo lo sviluppo di questa scena. In mezzo al gioco alternato fra il sole e l'ombra, fra il bianco e il nero, nella pittura pavesiana, spicca il colore dei mattoni del pavimento, sono rossi. L'interno rosso del pavimento del salotto, in corrispondenza con quello del terrazzo (le mattonelle di terraccotta hanno lo stesso colore), si oppongono visualmente di nuovo al bianco delle lenzuola, appena lavate e stese all'aperto, sul terrazzo.

La luminosità del bianco, all'esterno sul terrazzo, è rafforzata ancora, oltre che dalle campiture dei panni $\operatorname{stesi}^{10}$, anche grazie al riferimento all'asciugamano di Irene e il colore dorato dei suoi capelli biondi che, come le lenzuola, si stanno asciugando al sole. Questi due centri di bianco, sul terrazzo, condensano la luce solare della scena e la riflettono, raddoppiandone così la forza visiva.

Si deve tener conto, però, che il quadro, a partire dall'ultimo motivo figurativo scelto per la sua chiusura, la chèvelure, allarga le sue suggestioni sensuali, muovendosi stilisticamente tra il classicismo e la modernità (Borney 1994: 14-15). La "pettinatura" di Irene e i suoi capelli biondi, asciugati al sole, raccolgono, dunque, tanto la forza sensuale delle scene mitologiche e dei quadri del Rinascimento e del Classicismo quanto i suggerimenti sensuali dell'erotismo poetico decadente ${ }^{11}$.

Questo passaggio visuale di Anguilla, dal sole all'ombra e dall'ombra al sole, raggiunge in questo modo tutta la sua forza di bellezza sensuale, grazie all'implicito e al non detto nel testo ${ }^{12}$. L'esempio si muove, dunque, all'interno dei parametri visivi del non finito e dello studio dell'effetto luce. I colori pavesiani non sono descritti, ma, come sappiamo già, sono messi in gioco attraverso l'allusione, coinvolgendo così tutte le possibilità ermeneutiche che ogni colore e ogni oggetto della scena comportano.

${ }^{10}$ Sembra opportuno ricordare adesso i lavori di Giuseppe Pelizza da Volpedo (18681907) dedicati, appunto, allo studio dell'effetto luce della biancheria al sole, uno dei motivi pittorici ricorrenti all'interno dell'opera del grande pittore del secondo Ottocento italiano (Damigella 1981; Scotti 1986: scheda 899).

${ }^{11}$ Baudelaire fa dei capelli neri dell'amante il simbolo assoluto di ogni poesia e sensualità. Ma dobbiamo tener conto che i capelli di Santina, descritti ora in modo indiretto, come quelli di Irene, dell'esempio successivo, sono biondi. Di conseguenza, la bellezza di queste due ragazze, le bionde del romanzo, riproduce più da vicino il canone petrarchesco e rinascimentale; cioè, quello classico. La pettinattura delle donne costituisce un motivo ricorrente all'interno dell'arte occidentale, ed è anche presente nell'impressionismo francese, per esempio in Degas o in Renoir.

${ }^{12}$ Se i capelli costituiscono un centro erotico e feticistico per eccellenza, anche le lenzuola stirate sul terrazzo hanno, in questo caso, delle chiarissime connotazioni sessuali (Bornay 1994). Di nuovo qui è l'associazione analogica per contiguità la responsabile dei valori simbolici, marcatamente erotici, del testo. 
Anguilla, da povero garzone scalzo al servizio della famiglia di sor Matteo, entra forse per la prima volta in un «luogo unico», nella casa delle signorine. Si introduce così nel cuore stesso del profumo sensuale delle ragazze (che può intravvedere, ma mai toccare). Egli deve fare un doppio movimento: entrando dal "fuori" del pieno sole estivo, deve attraversare l'oscurità della stanza per arrivare di nuovo al sole, dove ha luogo la «visione» dell'intimità domestica e femminile. Irene si fa asciugare i capelli, probabilmente soltanto coperta a metà, ed è lì, in piena luce, che la biancheria e l'asciugamano della ragazza, lavati e svolazzanti all'area aperta, portano al protagonista i primi sentori di un'intimità erotica a lui negata, e allora nemmeno del tutto sospettata.

Nell'ultimo brano scelto, si assiste anche a un bagno: il bagno di Irene e Santina (le due bionde) in una giornata estiva. Nascosto dietro il sambuco, Anguilla, come un voyeur, guarda la bellezza delle due sorelle, in armonia con quella della luce e del paesaggio ${ }^{13}$.

Ma un giorno che Irene era venuta a far giocare Santina nella sabbia e non c'era nessuno, le vidi correre e fermarsi nell'acqua. Stavo nascosto dietro un sambuco. La Santina gridava mostrando qualcosa sull'altra riva. E allora Irene aveva posato il libro, s'era chinata, tolte le scarpe e le calze, e così bionda, con le gambe bianche, sollevando la gonna al ginocchio, era entrata nell'acqua. Traversò adagio, toccando prima col piede. Poi gridando a Santina di non muoversi, aveva raccolto dei fiori gialli. Me li ricordo come se fosse ieri (Pavese 2008: 639).

È chiaro che Irene, in questa descrizione, riprende anche la sensualità e l'erotismo delle figure della mitologia classica. Nella scena de La luna si trovano presenti tutti gli elementi necessari per parlare del bagno di una ninfa, di una dea, del bagno di Diana, o persino, già all'interno della tradizione ebraica e cristiana, del bagno di Susanna. Siamo nuovamente davanti a un topos letterario e pittorico ricorrente in tutte le manifestazioni artistiche occidentali ${ }^{14}$.

La forza visiva di quest' ekphrasis, essendo anche una scena en plein air, è incentrata principalmente sulla potenza sensuale della luce e dei suoi riflessi sui colori chiari: l'azzurro del cielo e dell'acqua che possiamo indovinare, il bianco della carnagione della ragazza, il dorato luminoso dei suoi capelli e dei fiori da lei stessa raccolti. All'interno di questo ventaglio di colori tendenti sul caldo, due soltanto ne sono rilevati, cioè nominati: il bianco delle gambe di Irene e il giallo dei fiori. Seguendo il ragionamento fatto negli altri esempi studiati, possiamo, quindi, dedurre che queste due tinte, esplicitamente citate nel testo, ricevano una maggiore

${ }^{13}$ Ernesto Treccani, nel 1987, nella sua testimonianza al Congresso di San Salvatore dedicato a Pavese, intitolata Come ho dipinto «La luna e i falò», citava già questo stesso passaggio del romanzo del '50 (Treccani 1987: 282).

${ }^{14}$ Dalla pittura fiamminga nel XVI secolo, con Marten Van Heemskerk che dipinge il bagno di Susanna, passando da Artemisia Gentileschi, Tintoretto, Rubens, Rembrant, lo stesso motivo femminile del bagno si può trovare in Felice Carrera, Carlo Carrà, Giorgio De Chirico, Renato Gottuso e lo stesso Carlo Levi. 
intensità. Gli altri ci offrono la possibilità di immaginare il colore, ma in questo caso il bianco e giallo sono "dipinti" direttamente dallo scrittore, acquistando una completa potenza visiva.

In questo ultimo quadro pavesiano, appaiono anche, sempre tramite la menzione indiretta, il verde e il giallo dei fiori del sambuco, e soprattutto il dorato del sole luminoso e intenso che trasforma tutta la scena in una «visione» pardisiaca e idillica (dantesca?). Siamo indubbiamente agli antipodi della durezza della vita delle donne di Gaminella.

D'altra parte, sembra necessario insistere sulla sensualità classica del quadro de La luna, descritto come al rallentatore. Se è vero che il testo è movimentato all'inizio dalla corsa di Santina verso il ruscello, questa velocità è subito interrotta. La presenza dei sintagmi verbali «fermarsi all'acqua» e «aveva posato il libro», detto dell'azione delle due sorelle, prima di entrare nel fiume, insieme all'utilizzo di «traversò adagio», ci parla proprio del movimento tranquillo ed armonioso della scena.

Tutta l'azione centrale si svolge davanti agli occhi di Anguilla in un ritmo rallentato. Si osservi anche in questo senso come la serie di proposizioni parattattiche legate fra di loro in una serie enumerativa lunga, di cinque membri, lascia solo alla fine il centro semantico dell'enunciato: «E allora Irene aveva posato il libro, s'era chinata, tolte le scarpe e le calze, e così bionda, con le gambe bianche, sollevandosi la gonna, era entrata nell'acqua», che contribuisce ancora di più a questa lentezza dell'azione testuale. Irene si toglie le scarpe e le calze, per eccellenza simboli erotici feticistici della femminilità, in silenzio. Mentre il protagonista contempla assorto il quadro di bellezza sensoriale che conserverà nella sua prima memoria erotica per tutta la vita.

D'altronde pare opportuno ricordare che la scena appena analizzata, nella sua potenza visiva, intrattiene uno stretto rapporto con la poesia Passerò per Piazza di Spagna, composta il 28 marzo 1950, e dedicata a Constance Dowling. In essa il protagonismo della luce solare del mattino, riflettuta sulle acque della fontana di Roma, in armonia con il biancore della donna «sarai tu - ferma e chiara», ci parlano un'altra volta della maestà deificata della figura femminile del testo ${ }^{15}$, e della deificazione sacrale dell'ultimo amore tragico di Pavese, quello che lo porterà definitivamente verso la morte, dopo «aver lavorato e aver dato poesia agli uomini [...]» (16 agosto 1950; Pavese 2000: 399).

\section{A modo di «bozzetti» conclusivi}

Dopo tutto quanto è stato detto fin qui, con i testi dello scrittore alla mano, siamo in grado di affermare che contrariamente a quanto è stato detto dalla critica d'arte più recente (Lamberti 2007: 41), Pavese era attentissimo alla visibilità degli oggetti

${ }^{15}$ Il paesaggio della Roma più dannunziana, non solo lascia vedere le tracce del poeta di Pescara nel testo di Pavese, bensì ci parla ancora una volta della visione pittorica pavesiana alla ricerca dell'eterna "Bellezza Femminile" (Gigliucci 2008). 
e dei luoghi da lui frequentati, principalmente quelli che formavano parte del suo serbatoio immaginario infantile e adolescenziale.

Siamo dell'avviso, in funzione dell'analisi portata avanti in questo lavoro, che se è vero che lo scrittore predilige l'evocazione e la suggestione verbale (Beccaria 2000: XVI; Lamberti 2007: 41) (è un fatto innegabile), non è meno certo che, come lo stesso autore afferma nel suo Diario, all'interno della sua creazione letteraria, le immagini-simbolo e i loro colori costituiscano una rete testuale molto produttiva dal punto di vista ermeneutico.

D'altra parte siamo dell'avviso che gli esiti di sensorialità visiva raggiunti da tanti componimenti pavesiani siano concomitanti alle grandi esperienze pittoriche contemporanee, iniziate dall'impressionismo e continuate dal post-impressionismo. Tale fatto non pone in contraddizione il possibile rapporto di Pavese con il gruppo degli amici artisti torinesi: Nicola Galanti, Felice Casarotti, Giulio da Milano, Piero Martina ed altri, citati da Lamberti (2007: 42-47); molti di questi senz'altro continuatori delle grandi imprese d'innovazione artistica aperte dagli impressionisti ${ }^{16}$.

Ammettendo che la vicinanza di questi pittori torinesi contemporanei al nostro autore possa aver facilitato la familiarità di Pavese con l'esercizio della pittura (a parte il saldo legame con il primo Sturani, di cui abbiamo già parlato nella prima parte di questo studio), riteniamo che la marcata insistenza visiva dei suoi testi più poetici affondi principalmente le sue radici nella letterattura stessa, e principalmente nella poesia. Come abbiamo già detto, in Guido Gozzano, in Charles Baudelaire, in Walt Whitman, e anche in Gabriele D'Annunzio ${ }^{17}$.

Ma tornando a Whitman, non dobbiamo dimenticare che il poeta americano era stato profondamente ammirato da Enrico Nencioni e dallo stesso Carducci. Il critico d'arte fiorentino, in una delle sue Discussioni artistiche, nel 1876, aveva già stabilito un incisivo nesso stilistico di soluzioni formali, poetiche e visive fra Whitman e Courbet. «[...] il soffio inmenso e la potente originalità whitmaniana!. [... ] Sai che cosa è il Whitman? - È il Courbet della poesia?» (Balloni 2007: 89).

La citazione precedente potrebbe servire da solida giustificazione per mettere ulteriormente a fuoco il legame fra la pittura degli inizi dell'impressionismo francese e l'opera del poeta americano. E l'autore di Foglie d'erba, come è ben noto, riposa alla base della formazione accademica e letteraria di Cesare Pavese. Lo scrittore italiano trovò sicuramente nelle pagine dell'americano la suggestiva forza vitale e visiva che avrebbero anche sviluppato, fino alle sue ultime conseguenze, gli impressionisti francesi e italiani, e i pittori del primo Novecento.

Ma a parte la traccia whitmaniana in Cesare Pavese, non dobbiamo sottovalutare l'ipotesi relazionale fra la figuratività italiana tardo ottocentesca e la visività pavesiana. Sembra più che probabile l'esistenza di un rapporto, forse inconsapevole da parte dello stesso Pavese, tra la marcata potenza delle sue immagini e la pittu-

${ }^{16}$ Felice Cesarotti era il presidente della Società delle Belli Arti Antonio Fontanesi e fu il responsabile della mostra fatta a Lorenzo Delleani a Torino nel 1925 (Mantovani 2008: 57).

${ }^{17}$ Già Marziano Guglielminetti (1998: xii) aveva mostrato la forte influenza di D'Annunzio sulla poesia di Pavese. Nella stessa linea Costa (1987: 147-158). 
ra del suo tempo, soprattutto di quella più direttamente influenzata dagli impressionisti. Il ruolo centrale che gioca lo studio della luce all'interno dell'esercizio artistico di entrambe le realtà di creazione (a parte il sostrato mitico e antropologico volutamente presente in Pavese ${ }^{18}$ ) ci si mostra anche come un nesso di unione importante fra le esperienze ekphrastiche dello scrittore e la sua ammirazione visiva verso le più famose realtà pittoriche dei suoi anni infantili.

Arrivati a questo punto, sembra opportuno ricordare in proposito la scuola dei pittori piemontesi della seconda metà dell'Ottocento. Molti degli artisti di Rivara (soprattutto dopo il 1881) si mostrano come veri propulsori del rinnovamento figurativo dell'arte italiana fra Otto e Novecento (Marini 2008:13-39). Pittori piemontesi e liguri come Lorenzo Delleani (1840-1908), Carlo Pittara (1835-1891), Ernesto Rayper (1840-1873), Enrico Reycend (1855-1928), o lo stesso Giuseppe Pelizza da Volpedo (prima citato), furono già considerati «poeti del vero», e «riscoperti» da Roberto Longhi nel 1952 (Marini 2008: 158). Oggi, dopo la quanto meno grave disattenzione occorsa in decadi precedenti da parte dei critici e degli intellettuali italiani ${ }^{19}$, questi artisti vengono considerati come dei veri maestri della pittura europea dell'epoca (Marini 2008; Maggio Serra 2008; Magnetti 2008; Mantovani 2008). Le loro prime esperienze artistiche sono servite come modello ai lavori delle posteriori generazioni dei pittori piemontesi e torinesi, già nel Novecento ${ }^{20}$.

Non dobbiamo dimenticare che la fama degli artisti della seconda metà dell'Ottocento italiano fu enorme durante gli anni dell'adolescenza di Pavese ${ }^{21}$. Di conseguenza, non sembra troppo azzardato pensare che molte delle stampe e degli almanacchi dei primi anni della vita del nostro scrittore siano state riproduzioni dei quadri più importanti di questi grandi maestri italiani del XIX secolo. La poderosa innovazione dei lavori di un Delleani e il suo realismo, o la poesia paesistica di un Reycend, lo studio dell'effetto luce di un Pelizza da Volpedo, per esempio, potrebbero trovarsi persino fra alcune delle pitture più ammirate dallo stesso Pavese ${ }^{22}$, soprattutto nei suoi anni giovanili ${ }^{23}$.

${ }^{18}$ Per un ulteriore approffondimento sulla visività poetica pavesiana in linea mitica ed antropologica (Mesiano 2009: 228-41).

${ }^{19}$ Mario Soldati fu uno degli intellettuali italiani che inizialmente non ebbero in considerazione il lavoro pittorico degli artisti piemontesi (Mantovani 2008: 56)

${ }^{20}$ Lo stesso lavoro di Cesarotti che in un principio si pensava solo continuatore dell'opera di Cézanne, oggi può essere visto, invece, sotto l'influenza della scuola piemontese di pittura, a cavallo fra Otto e Novecento (Mantovani 2008: 59).

${ }^{21}$ In concreto dobbiamo tener conto che a Lorenzo Delleani (1840-1908) furono dedicate a Torino due importanti mostre nella prima metà del secolo scorso; la prima nel 1925 a Palazzo Bricherasio e la seconda nel 1940 al Salone de «La Stampa». Fatto che ci parla della popolarità del pittore nella Torino di Cesare Pavese (Marini 2008: 14-15).

22 Tutte le famiglie nobili o borghesi di Torino della fine dell'Ottocento e dell'inizio del Novecento avevano un Delleani a casa. Tale dato ci parla dell'enorme popolarità del pittore (Magnetti 2008: 51).

${ }^{23}$ Guglielminetti notava già la tendenza di Pavese «alla fissazione di luoghi e persone e gesti grevi». Il critico parla della possibile influenza del Picasso del «periodo blu» sull'ope- 
Nei pittori ottocenteschi su citati, troviamo la forza impressionista e sensoriale del colore, il pregio poetico del non finito, la ricerca della bellezza del vero e dell'essenziale, lo studio poetico del paesaggio, ma anche la loro impegnata e attenta sensibilità figurativa verso il lavoro, le abitudini e la durezza della «vita dei campi». Nei loro quadri, la vendemmia, la raccolta, la semina, i lavori domestici, i quadri e le figure d'epoca aprono la strada verso una mistura tematica e stilistica a cavallo fra il tardo romanticismo e il realismo, fra l'impressionismo simbolista e decadente e il realismo poetico più irrazionalmente tragico e mitico, molto vivacemente presente nella migliore letteratura europea del Novecento.

In sostanza, possiamo chiudere questo lavoro dicendo che indubbiamente le strade che Pavese dovette percorrere fino alla scoperta della rivelazione simbolica dell'immagine poetica e dell'attimo statico e contemplativo furono necessariamente variegate e plurali. Ma, secondo quello che abbiamo tentato di dimostrare in questo lavoro, la potenza visiva della sua opera ci si mostra come una realtà chiara ed evidente; e questa ha indubbiamente molto di pittorico.

Di conseguenza, la nostra ipotesi è questa: se i grandi poeti del secondo Ottocento francese (Baudelaire), italiano (D'Annunzio ${ }^{24}$ ) ed americano (Whitman) sono alla base dell'esercizio letterario dello scrittore delle Langhe, non sembra del tutto sbagliato pensare che all'interno della visibilità pavesiana ci siano anche, in un modo forse non del tutto cosciente, insieme alle tendenze dell'arte novecentesca, i lavori più emblematici dei pittori italiani delle generazioni precedenti.

Questa nostra ipotesi si offre, ad ogni modo, come punto di partenza e di stimolo per nuove ricerche interdisciplinari sull'importanza dell'immagine visuale nella scrittura pavesiana; non abbiamo, dunque, la minima pretesa di offrire questi possibili legami artistici e letterari come una conclusione definitiva. Forse perché, come diceva Antonio Machado, siamo ben consapevoli di un piccolo grande fatto: solo «se hace camino al andar». Siamo, invece, assolutamente certi del peso determinante che la visione e la contemplazione dei «luoghi unici» hanno avuto nella visività pittorica di Pavese. Questa costituisce il centro mitico del fare e dell'essere pavesiano. La poetica visiva dello scrittore, agendo, quindi, dall'interno stesso del suo immaginario mitico, approda inesorabilmente alla rivisitazione memoriale e visuale degli «spazi sacri», quelli che vengono fissati staticamente con gli occhi interni di ogni poeta nel quadro, nella pittura e nella sua corrispondente ekphrasis di poderosa bellezza elegiaca.

ra del nostro poeta (Guglielminetti 1998: XIV). Secondo quello che è stato detto fin qui, soprattutto in ragione dell'attento studio pavesiano sull'effetto luce e sulla vivacità simbolica dei colori, sempre usati in modo contrastivo, pensiamo che siano gli impressionisti francesi insieme ai piemontesi del secondo Ottocento (e anche ai toscani, fondamentalmente nei loro lavoro paesistici, e non tanto in quelli a tema storico) quelli che presentano una sensibilità visiva più affine a quella di Cesare Pavese nei suoi migliori frammenti ekphrastici.

${ }^{24}$ Non dobbiamo dimenticare che D'Annunzio era molto amico di Paolo Michetti (18511929), uno dei pittori pescaresi la cui esperienza figurativa è legata al gruppo di artisti italiani napoletani di questa stessa epoca. 
Vogliamo precisare ancora una volta che in questo lavoro, abbiamo studiato soltanto pochi casi della visibilità poetica dell'autore, si potrebbero trovarne tanti altri da scrivere sicuramente un'intera monografia ${ }^{25}$. Questa nostra diversa e, forse non "ortodossa" interpretazione dell'opera pavesiana, è partita dai testi, dalla nostra attenta, ripetuta e umile lettura; soprattutto dalla nostra più commossa famigliarità con La luna e i falo; ormai una nostra compagna di strada.

Nell'origine di questo lavoro c'è anche una personale ricognizione poetica, dal vivo, della terra pavesiana. Il Piemonte, Torino, le Langhe e il Monferrato del XXI secolo, diventati altri riguardo ai passati scenari pavesiani, nonostante questa abissale differenza, ci si sono rivelati come antichi spazi carichi di poesia e di bellezza, malgrado le più recenti storie di vite rurali, marcate dalla miseria, dallo sfruttamento, dalla violenza e dall'ingiustizia della Storia. Grazie alla luce dorata e ai colori delle terre subalpine, soprattutto nella bella stagione, attraverso la loro ondulante soavità collinare, attraverso le vigne e le piante, i loro fiumi e i loro colori, grazie ai campi piemontesi, che sono e non sono quelli di una volta, non soltanto ho rivisto le scene de La luna e i falò, bensì mi è stato concesso di ricordare le estati di infanzia nelle terre verdi del nord della Spagna, fra le montagne, nei paesi della Cantabria.

In questi ultimi dieci anni, quando, come italianista ho piantato finalmente le mie radici italiane in Piemonte, grazie alle Langhe e al Monferrato, grazie anche all'ospitalità degli amici piemontesi, grazie a Pavese e grazie ai suoi quadri più rappresentativi, anch'io ho potuto rivedere, attraverso l'occhio netto dell'arte e della letteratura italiana, le migliori immagini del mio (forse) miglior tempo passato.

\section{Bibliografia}

ADORNo, Theodor W. (1970): Teoria estetica. Torino, Einaudi.

Ballon, Silvio (2007): «Telemaco Signorini, i Macchiaioli e gli ambienti letterari del tempo», in Francesca Dini (2007), pp. 81-94.

BÀrberi-SQuAROtTI, Giorgio (1964): «Pavese o la fuga nella metafora», in Sigma, 3/4, pp. 165-187.

BÀRBERI-SQUAROTTI, Giorgio (1987): «Il viaggio come struttura del romanzo pavesiano», in Giovanna Ioli (1987), pp. 47-62.

BASILE, Bruno (2003): «La "finestra" di Pavese: analisi di un'immagine ossessiva», in $L a$ finestra socchiusa. Ricerche tematiche su Dostoevskij, Kafka, Moravia e Pavese. Roma, Salerno Editrice, pp. 503-518.

${ }^{25}$ Non abbiamo toccato i notturni pavesiani dedicati alle Langhe per ragioni di tempo e di spazio. Ma dobbiamo tener conto che, dallo stesso titolo del romanzo del '50: La luna e $i$ falò, possiamo già vedere il bellissimo contrasto coloristico fra l'oscurità della notte, la luce lunare e il rosso del fuoco dei falò. In questa intensità coloristica e luminosa vale la pena rilevare il momento tragico dell'incendio provocato da Vallino in Gaminella; anche in questo caso prevale il contrasto marcatissimo fra il nero del notturno e il rosso del fuoco: «Riflessi rossi morivano a piede del muro, sprigionando una fumata nera» (Pavese 2008: 663). 
BeCCARIA, Gian Luigi (2000): «Introduzione» a Cesare Pavese, La luna e i falò. Torino, Einaudi, pp. v-xxx.

BORNAY, Erika (1994): La cabellera femenina. Madrid, Cátedra.

Bossetti, Gilbert (1960): «La poétique du mythe de l'enfance de Pavese», Cahiers du Cercic, 16, pp. 143-187.

CostA, Simona (1987): «Pavese e D’Annunzio», in Giovanna Ioli (1987), pp. 147-158.

DAmigella, Anna M. (1981): La pittura simbolista in Italia 1885-1900. Torino, Einaudi.

DINI, Francesca (a cura di) (2007): I Macchiaioli. Sentimento del vero. Torino, Silvana Editoriale.

DINI, Francesca (2007); «Poesia dei Macchiaioli» in Francesca Dini (2007), pp. 15-45.

Gionnola, Elio (1971): Cesare Pavese. La poetica dell'essere. Milano, Marzorati, Editore.

GioAnola, Elio (2002): «Introduzione» a Cesare Pavese, Feria d'agosto. Torino, Einaudi, pp. v-xxii.

Gionnola, Elio (2003a): Cesare Pavese: la realtà, il silenzio, l'altrove. Milano, Jaka Book.

Gionnola, Elio (2003b): «Pavese e il silenzio», Cuadernos de Filología Italiana, 9, pp. 121-138.

Gombrich, Ernest H. (1997): Gombrich Esencial. Textos escogidos sobre arte y cultura. Barcelona, Debate.

Guglielminetti, Marziano (1990): Una poetica “tenzone”: Mario Sturani e Cesare Pavese, in M. Mimita Lamberti (a cura di). Torino, Allemandi.

Guglielminetti, Marziano (1998): «Introduzione» a Le poesie di Cesare Pavese. Torino, Einaudi, pp. v-xvii.

Gigliucci, Roberto (2001): Cesare Pavese. Torino, Einaudi.

Gigliucci, Roberto (2008): «Pavese (e D’Annunzio) a Piazza di Spagna». <www. disp. let.uniroma1.it/fileservices/.../>

Ioli, Giovanna (a cura di) (1987): Cesare Pavese oggi. Atti del Convegno Internazionale (S. Salvatore Monferrato, 25-27 settembre). S. Salvatore Monferrato.

LAMBERTI, M. Mimita (2007): «La Torino dei pittori e la città di Pavese», in Mariarosa Masoero (2008), pp. 37-47.

LAVEZZI, Gianfranca (2004): Breve dizionario di retorica e stilistica. Roma, Carocci.

MAGGIO SERRA, Rosanna (2008): «Delleani e la responsabilità della critica», in Giuseppe Luigi Marini (2008a), pp. 41-48.

MagnetTi, Daniela (2008): «L'impressione del paesaggio tra verde piemontese e grigio ligure di fine Ottocento», in Giuseppe Luigi Marini (2008a), pp. 49-54.

Mantovani, Pino (2008): «1925, Palazzo Bricherasio. Un’occasione per pensare Delleani in chiave "moderna"», in Giuseppe Luigi Marini (2008a), pp. 55-61.

MARINI, Giuseppe Luigi (a cura di) (2008a): Delleani e il suo tempo. Milano, Silvana Editoriale.

MARINI, Giuseppe Luigi (2008b): Biografie, in Giuseppe Luigi Marini (2008a), pp. 225-235.

MARTíNEZ GARRIDO, Elisa (2010): «Del “instante estático”, del “estado de gracia” y de la experiencia sensorial en Cesare Pavese: ¿ecos y reflejos de pinceladas proustianas?», in Lourdes Carriedo y $\mathrm{M}^{\mathrm{a}}$ Luisa Guerrero (eds.), Marcel Proust: ecos y reflejos. BruxelesParis-Berlin, Peter Lang, pp. 315-350.

Masoero, Mariarosa (a cura di) (2008): Cesare Pavese e la "sua” Torino. Torino, Lindau.

MAsoero, Mariarosa e ZACCARIA, Giuseppe (a cura di) (2008): Cesare Pavese, I capolavori. Torino, Einaudi, «Biblioteca della Pléiade».

Mengaldo, Pier Vincenzo (2005): Tra due linguaggi. Arti figurative e critica. Torino, Bollati Boringhieri. 
Mesiano, Luisiella (2009): Il ritratto oscurato di Pavese allegro. Lettura e documenti di un'inedita condizione espressiva. Milano, Officina Libraria.

MilA, Massimo (1985): "Presentazione della Mostra "Homage a Cesare Pavese"». $<$ www.internetculturale.it $>$.

Mondo, Lorenzo (1964): «Fra Gozzano e Whitman: le origini di Pavese», in Sigma 3-4, 1964, pp. 3-21.

Pavese, Cesare (1966a): Lettere 1924-1940, vol. I, a cura di Lorenzo Mondo. Torino, Einaudi.

Pavese, Cesare (1966b): Lettere 1945-1950, vol. II, a cura di Italo Calvino. Torino, Einaudi.

PAVESE, Cesare (1998): Le poesie, a cura di Mariarosa Masoero. Torino, Einaudi.

PAvese, Cesare (2000): Il mestiere di vivere, a cura di Marziano Guglielminetti e Laura Nay.Torino, Einaudi.

PAvese, Cesare (2002): Feria d'agosto, a cura di Elio Gioanola. Torino, Einaudi.

PAVESE, Cesare (2006): Tutti i racconti, a cura di Mariarosa Masoero. Torino, Einaudi.

PAVESE, Cesare (2008): La luna e i falò, in Mariarosa Masoero e Giuseppe Zaccaria (a cura di), pp. 563-687.

ScotTI, Aurora (1986): Pelizza da Volpedo. Catalogo generale. Milano, Electa, Scheda 899. TreCCANI, Ernesto (1987): Come ho dipinto «La luna e i falò», in Giovanna Ioli (1987), pp. 281-282.

VACCANEO, Franco (1999): Cesare Pavese. Cavallermaggiore, Gribaudo. 